\title{
EFFECT OF HEAT TREATMENT ON CYCLIC FATIGUE, FLEXIBILITY AND PHASE TRANSFORMATION OF TWO CONTROLLED MEMORY NITI ROTARY INSTRUMENTS
}

\author{
Wafaa A. Khalil *
}

\begin{abstract}
Aim: The aim of this study the effect of heat treatment on flexibility, cyclic fatigue and of OneCurve nickel-titanium (Ni-Ti) rotary file (OC) and compare them with those of EdgeFile (EF) NiTi file as both files manufactured from a controlled memory wire.

Methods: A sample of 45 instruments with tip size of 25 and 0.06 taper from both OC and EF were selected for the current study. The cyclic fatigue testing was performed using dynamic mode. The bending resistance was tested using the universal testing machine and files were bent until reach $45^{\circ}$. In addition, dispersive scanning calorimetric analysis (DSC) was carried out for identification of $\mathrm{A}_{\mathrm{f}}$ transformation temperature. An independent $t$-test was used in the statistical analysis and significance difference was set at p-value 0.05 .
\end{abstract}

Results: The EF files showed significantly greater resistance to cyclic fatigue $(\mathrm{p}<0.05)$. As well as, the EF exhibited less bending resistance than OC $(p<0.5)$. The DSC analysis showed that the OC has an $\mathrm{A}_{\mathrm{f}}$ near $54^{\circ} \mathrm{C}$, while the $\mathrm{EF}$ was near $57^{\circ} \mathrm{C}$.

Conclusions: The EdgeFile NiTi rotary instruments have high flexibility and high resistant to cyclic fatigue than OneCurve does when tested in dynamic mode and both have a close has an $\mathrm{A}_{\mathrm{f}}$ temperature exceeding $50^{\circ} \mathrm{C}$

\section{INTRODUCTION}

Nickel-titanium (NiTi) rotary instruments represent an evolution in optimizing preparation of a root canal system. The main drawback of $\mathrm{NiTi}$ instruments is their sudden fracture, which could complicate and compromise the out come of endodontic treatment ${ }^{(1)}$.
Manufacturers of NiTi rotary instruments have been inventing methods that improve the available wide range instruments in consequence with introducing new instruments with improved designs and kinematics ${ }^{(2,3)}$. Recently the focus of the manufacturer was directed to altering the microstructure of the NiTi with heat treatment

* Associate Professor, Department of Endodontics, Faculty of Dentistry, King Abdulaziz University, Jeddah, Saudi Arabia 
and cooling treatment. The different stages of heat treatment and rate of cooling improved the instrument flexibility and cyclic fatigue resistance. A wide variety of NiTi wires were produced by thermomechanical treatment of NiTi alloy like M-wires, Max, R-phase, controlled memory (CM) wires and recently $\mathrm{C}$-wire. Instruments made from controlled memory wire were 300\%-800\% more resistant to cyclic fatigue than regular NiTi wires ${ }^{(4)}$. Moreover, instruments manufactured from a heattreated NiTi wires exhibit an increased austenite transformation temperature and significant change in the phase transformation ${ }^{(5)}$.

The EdgeFiles (EF X3, EDGEENDO, Albuquerque, NM, USA) is a recently developed electropolished rotary system made of an annealed heat-treated NiTi alloy called Fire-Wire that does not have the shape memory property. The file is has a parabolic cross section and a constant taper of 06. The EdgeFile rotary system showed the lowest transportation when used to prepare the mesiobuccal root canal of mandibular molar compared to WaveOne and Reciproc ${ }^{(6)}$. When testing the cyclic fatigue of EF under different temperature, the NCF was the highest for the EF group followed by the Vortex Blue group and lowest for the ESX Brassler group ${ }^{(7)}$.

One Curve (OC) is a new NiTi rotary instruments fabricated from a novel heat-treated $\mathrm{C}$-wire launched in February 2018 (Micro-Mega, Besancon, France). The C-wire is a heat-treated pre-bendable and controlled memory wire. These new instruments are a single use files with variable cross-section along the blade. The first zone presents a variable 3 -cutting edge, while the second has a cross section that progressively converted to 2 cutting edges with the same geometric features as OS.

Therefore, the aim of this study was to test the effect of heat treatment technique on the cyclic fatigue resistance, flexibility, and phase transformation for OneCurve and EdgeFile. The null hypothesis tested is there is no significant difference in the mechanical properties between the two files.

\section{MATERIALS AND METHODS}

A sample of 35 instruments size 25, .06 taper with $25 \mathrm{~mm}$ length from OC and EF rotary instruments were selected for this study after inspected for integrity and defects under the stereomicroscope.

\section{Cyclic fatigue testing}

A set of 15 new OC and EF files was tested for cyclic fatigue in the dynamic mode. A custommade device with a preset computer program was attached to a universal machine that guarantees a reproducible position for all instruments in the selected dynamic mode for testing the cyclic fatigue ${ }^{(8)}$. In the fatigue test, the instruments were rotated freely in an artificial stainless steel canal with $60^{\circ}$ curvatures and a $5-\mathrm{mm}$ radius with the curve beginning $5 \mathrm{~mm}$ from the tip. The inner diameter of the canal was designed to accommodate the size and taper of the tested instrument. The stainless steel plate was covered with tempered glass and filled with synthetic oil to prevent friction. Both OC and $\mathrm{EF}$ were tested at $300 \mathrm{rpm}$ and $2.5 \mathrm{~N} / \mathrm{cm}$ torque following the manufacturer's recommendations. A continuous axial oscillating movement was utilized at a frequency of 1 cycle/ $2 \mathrm{sec}$ (Hertz) total oscillation amplitude of $3 \mathrm{~mm}^{(9-10)}$. The fracture was observed visually through a magnifying lens and the time to fracture was recorded. The total time to fracture was recorded. The length of the fractured segments was measured using digital micro-calipers.

\section{Flexibility testing}

10 randomly selected instruments of each file were tested for flexibilityusing a universal testing machine (MutliTest 2.5-i, Mecmesin, Newton House, West Sussex, UK) in compliance with ISO 3630-1 specification (ISO 3630-1:2008). A preset computer program was attached to the universal testing machine, which guarantees a reproducible $45^{0}$ position for all instruments (Emperor, Mecmesin, Sinfold, UK). The test was performed using a $20-\mathrm{N}$ load at speed of $15 \mathrm{~mm} / \mathrm{min}$. instrument one end was attached to a flexible stainless steel wire fixed 
to the universal testing machine head and the $3 \mathrm{~mm}$ from the tip was secured to the machine ${ }^{(10)}$.

\section{Differential scanning calorimetric analysis (DSC)}

Two segments were obtained from the apical and middle parts of each file $(\mathrm{n}=10)$. The segments of 4-5 mm length were cut from each instrument by using a slow-speed diamond saw under watercoolant. Partial cycle of DSC analysis was performed over a temperature range of $-40^{\circ} \mathrm{C}$ to $80^{\circ} \mathrm{C}$ at a rate of $5^{\circ} \mathrm{C} / \mathrm{min}$ by using liquid nitrogen coolant at a rate of $50 \mathrm{ml} / \mathrm{min}$ (PYRIS, PerkinElmer Diamond Series DSC; PerkinElmer, Shelton, CT) ${ }^{(5,11)}$. The austenite-start (As) and finish $\left(\mathrm{A}_{\mathrm{f}}\right)$ temperature as well as, the martensite-start $(\mathrm{Ms})$ and finish $\left(\mathrm{M}_{\mathrm{f}}\right)$ temperature were specified ${ }^{(5)}$.

\section{Fractographic analysis}

The fractured segments ultrasonically cleaned in absolute alcohol and examined under the SEM for the characterization of the topographical features of the fractured surfaces at various magnifications $(150-1500 \mathrm{X})$ to determine the fracture mode (Quanta 250 FEG, FEI, Eindhoven, Netherlands).

\section{Statistical analysis}

The data for each test were analyzed statistically using the normality of which was the KolmogorovSmirnov test for determination of normality and an independent sample $t$-test to compare the OC and EF at significance level of $P<.05$ (SPSS v22 (SPSS, Inc., Chicago, IL).

\section{RESULTS}

The Kolmogorov-Smirnov test showed the data for the time to fracture were distributed normally. The EdgeFile instruments demonstrated significantly greater resistance to the fracture and high flexibility compared to OneCurve $(P<0.05)$, Table 1.

The DSC plots curves during heating and cooling showed that EF and OC have two peaks; the first one corresponded to the primary transformation from martensite to R-phase, while the second one corresponded to the transformation from the $\mathrm{R}$-phase to austenite. The $\mathrm{A}_{\mathrm{f}}$ of $\mathrm{OC}$ file was recorded at $54^{\circ} \mathrm{C}$ while $\mathrm{A}_{\mathrm{f}}$ of $\mathrm{EF}$ was at $57^{\circ} \mathrm{C}$ (Figure.1).

The longitudinal views of the fractured instruments showed similar aspect of no unwinding with abrupt fracture in the $\mathrm{OC}$, while the $\mathrm{EF}$ showed multiple cracks starting at the cutting edge (Figure.2). The topographic appearance of the fractured face showed crack originating areas at the cutting edge, crack propagation, and areas of dimples that represent the overload area. The EdgeFile showed multiple crack origin while OC showed one crack origin.

TABLE (1) Mean and standard deviation of the time to fracture, segment length, bending moment, and Differential Scanning Calorimetry.

\begin{tabular}{|c|c|c|c|c|c|}
\hline \multirow{2}{*}{ Group } & \multirow{2}{*}{$\begin{array}{c}\text { Time to fracture } \\
(\mathrm{Sec})\end{array}$} & \multirow{2}{*}{ Segment length } & \multirow{2}{*}{$\begin{array}{l}\text { Bending moment } \\
\text { (grm) }\end{array}$} & \multicolumn{2}{|c|}{ DSC analysis } \\
\hline & & & & $\mathrm{A}_{\mathrm{f}}$ Temperature & $\Delta \mathrm{H}(\mathrm{J} / \mathrm{g})$ \\
\hline OneCurve & $648 \pm 12.2$ & $2.51 \pm 0.17$ & $21.41 \pm 3.1$ & $54 \pm 31^{\circ} \mathrm{C}$ & $12.284 \mathrm{~J} / \mathrm{g}$. \\
\hline EdgeFile & $894 \pm 10.5$ & $3.12 \pm 0.210$ & $12.13 \pm 2.8$ & $57 \pm 23^{\circ} \mathrm{C}$ & $14.948 \mathrm{~J} / \mathrm{g}$. \\
\hline P-value & $P<0.0$ & $P>0.05$ & $P<0.05$ & $P>0.05$ & $P>0.05$ \\
\hline
\end{tabular}

All tested groups were significantly different from each others $(p<.05)$.

Time to fracture; M, mean; SD, standard deviation; N, Newton; DSC, Differential Scanning Calorimetry 


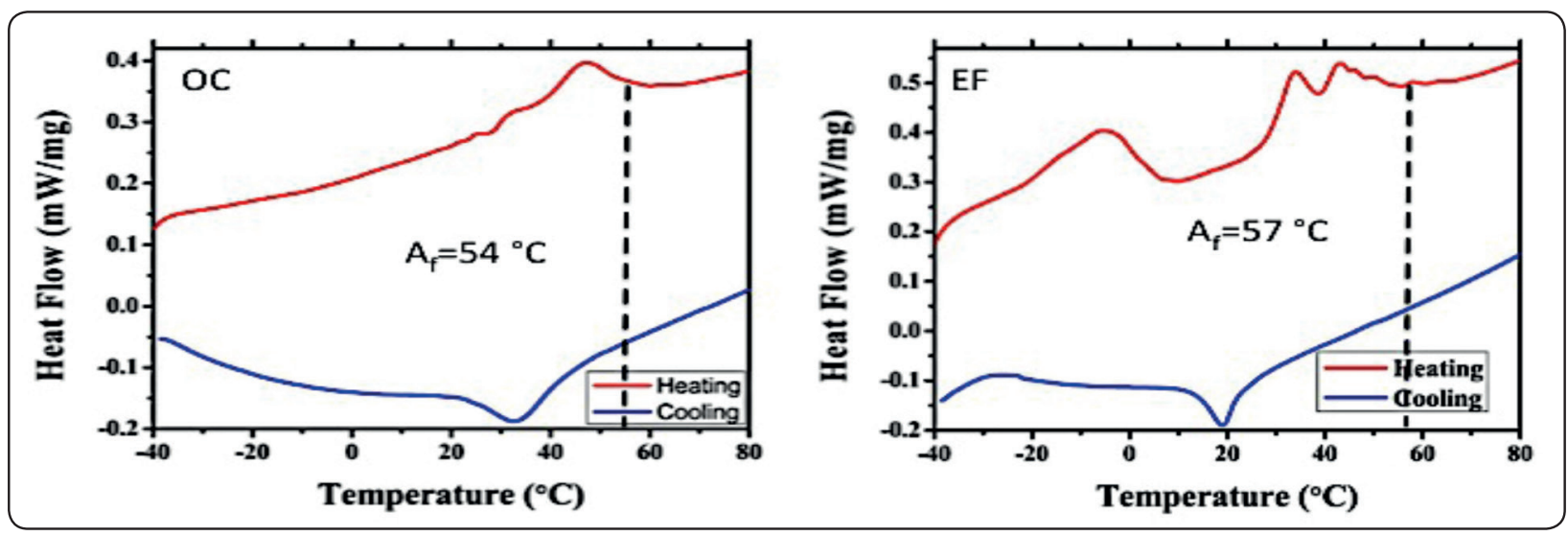

Fig. (1) The results of Differential Scanning Calorimetry analysis, (Left) OneCurve, (Right) EdgeFile
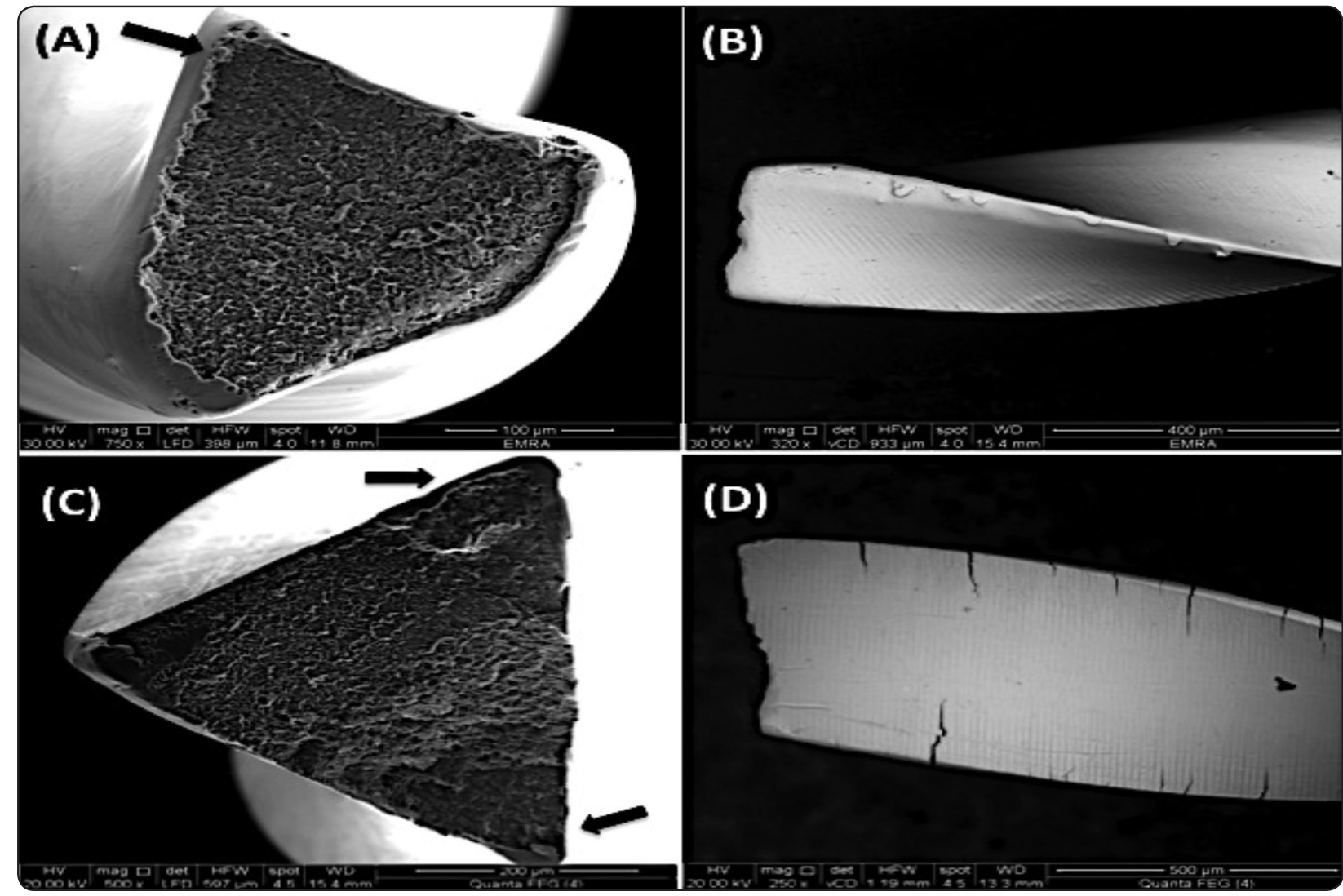

Fig. (2) The SEM micrograph of the lateral view and fracture face of the instruments after cyclic fatigue testing. (a) OneCurve fracture face showing one crack origin (arrow), (b) lateral view of OneCurve after testing showed no cracks, (c) EdgeFile fracture face showing multiple crack origin (arrow), and (d) lateral view of EdgeFile showing multiple cracks originating at the cutting edge. 


\section{DISCUSSION}

Instruments fracture during root canal preparation could happen suddenly and may compromise the therapeutic outcome ${ }^{(12)}$. The mechanical properties of the rotary instruments were tested in dynamic mode that is similar to clinical situation utilizing computer assisted mechanical testes. Both tested instruments are manufactured from controlled memory NiTi wires and have a triangular cross section.

Testing fatigue resistance of the NiTi rotary instruments has been studied extensively using the static mode in single and double canal curvature ${ }^{(13-14)}$, while other studies have used the dynamic mode ${ }^{(9-10,15)}$. The dynamic mode is the most suitable for testing the cyclic fatigue of continuous rotating instruments, as static mode does not reproduce the real clinical condition. Axial motions reduce the maximum stress caused by continuous tension and compression used in static mode, as the instrument remains longer in the straight part of the canal before it passes again through the high stress area. The pecking movement may be an essential factor in preventing the fracture of NiTi instruments in curved canals ${ }^{(16)}$.

Regarding the results of the current study, the EF instrument showed significantly greater resistance to fatigue and significantly higher flexibility than the OC did. Accordingly, the null hypothesis was rejected.

DSC analyses showed that, the EF and OC files had an $\mathrm{A}_{\mathrm{f}}$ temperature about $57^{\circ} \mathrm{C}$ and $54^{\circ} \mathrm{C}$ close to that of controlled memory files reported by previous study ${ }^{(11,17)}$. Previous studies reported that the $\mathrm{A}_{\mathrm{f}}$ temperature for typhoon files about $54^{\circ} \mathrm{C}$ and Vortex files $50^{\circ} \mathrm{C}$, which indicates that these files would be essentially in the martensitic phase at body temperature ${ }^{(5,11)}$. Instrument composed of martensite phase at room temperature have enhanced performance during root canal preparation ${ }^{(18)}$. This explains the remarkable long time to fracture and high flexibility compared to the results obtained by previous studies ${ }^{(4,19)}$. In summary, many previous studies reported an improvement of the cyclic fatigue resistance of the controlled memory instruments compared to the regular NiTi and m-wire which cam in agreement with our results ${ }^{(11,20-22)}$.

In the current study, both files exhibited long time to fracture. The significant difference between $\mathrm{EF}$ and $\mathrm{OC}$ regarding the fracture resistance and flexibility could be attributed to the method of thermal treatment, as EF is cryogenically tempered. According to Zhang et al 2012 the rate of cooling has a great influence on the phase transformation temperature and martensitic start and finish temperature which will decrease when shorten the rate of cooling ${ }^{(23)}$. Another factor can contribute in the cyclic fatigue resistance; the different cross sections of OC may act as a weakening factor that affects the fracture resistance ${ }^{(24)}$. Elnaghy and Elsaka (2018) reported that OC was not superior in cyclic fatigue resistance when tested in static mode compared to Vortex Blue used static mode and double curvature at $37^{\circ} \mathrm{C}^{(25)}$.

\section{CONCLUSIONS}

Within the limitations of this study, the EdgeFile NiTi rotary instruments have high flexibility and high resistant to cyclic fatigue than OneCurve does and both have a close has an $\mathrm{A}_{\mathrm{f}}$ temperature exceeding $50^{\circ} \mathrm{C}$

\section{REFERENCES}

1. Lin LM, Rosenberg PA, Lin J. Do Procedural Errors Cause Endodontic Treatment Failure? The Journal of the American Dental Association 2005;136(2):187-193.

2. Braga LCM, Faria SilvaAC, Buono VTL, de Azevedo Bahia MG. Impact of Heat Treatments on the Fatigue Resistance of Different Rotary Nickel-titanium Instruments. Journal of Endodontics 2014;40(9):1494-1497.

3. Higuera O, Plotino G, Tocci L, Carrillo G, Gambarini G, Jaramillo DE. Cyclic Fatigue Resistance of 3 Different Nickel-Titanium Reciprocating Instruments in Artificial Canals. Journal of Endodontics 2015;41 (6):913-5.

4. Shen Y, Qian W, Abtin H, Gao Y, Haapasalo M. Fatigue Testing of Controlled Memory Wire Nickel-Titanium Rotary Instruments. Journal of Endodontics 2011; 37(7):997-1001. 
5. Shen Y, Zhou HM, Zheng YF, Campbell L, Peng B, Haapasalo M. Metallurgical Characterization of Controlled Memory Wire Nickel-Titanium Rotary Instruments. Journal of Endodontics 2011;37(11):1566-1571.

6. Hasheminia SM, Farhad A, Sheikhi M, Soltani P, Hendi SS, Ahmadi M. Cone-beam Computed Tomographic Analysis of Canal Transportation and Centering Ability of Single-file Systems. Journal of Endodontics 2018;44(12):1788-1791.

7. Dosanjh A, Paurazas S, Askar M. The Effect of Temperature on Cyclic Fatigue of Nickel-titanium Rotary Endodontic Instruments. Journal of Endodontics 2017;43(5):823-826.

8. Rodrigues CS, Vieira VTL, Antunes HS, De-Deus G, Elias CN, Moreira EJL, Silva EJNL. Mechanical Characteristics of Counterfeit Reciproc Instruments: a Call for Attention. International Endodontic Journal 2017;51 (5): 556-563.

9. Yao JH, Schwartz SA, Beeson TJ. Cyclic Fatigue of Three Types of Rotary Nickel-Titanium Files in a Dynamic Model. Journal of Endodontics 2006;32( 1):55-57.

10. De-Deus G, Leal Vieira VT, Nogueira da Silva EJ, Lopes H, Elias CN, Moreira EJ. Bending Resistance and Dynamic and Static Cyclic Fatigue Life of Reciproc and WaveOne Large Instruments. Journal of Endodontics 2014;40(4):575-579.

11. Shen Y, Zhou H, Coil JM, Aljazaeri B, Buttar R, Wang Z, Zheng YF, Haapasalo. ProFile Vortex and Vortex Blue Nickel-Titanium Rotary Instruments after Clinical Use. Journal of Endodontics 2015;41( 6):937-942.

12. Alapati SB, Brantley WA, Svec TA, Powers JM, Nusstein JM, Daehn GS. SEM Observations of Nickel-Titanium Rotary Endodontic Instruments that Fractured During Clinical Use. Journal of Endodontics 2004;31(1):40-43.

13. Elnaghy AM, Elsaka SE. Laboratory Comparison of the Mechanical Properties of TRUShape with Several NickelTitanium Rotary Instruments. International Endodntic Journal 2017;50(8):805-812.

14. Gu Y, Kum K-Y, Perinpanayagam H, Kim C, Kum DJ, Lim SM, Chang SW, Ho Beak S, Zhu Q, Yoo YJ. Various HeatTreated Nicke-Titanium Rotary Instruments Evaluated in S-Shaped Simulated Resin Canals. Journal of Dental Sciences 2017;12(1):14-20.

15. Ray JJ, Kirkpatrick TC, Rutledge RE. Cyclic Fatigue of EndoSequence and K3 Rotary Files in a Dynamic Model. Journal of Endodontics 2007;33(12):1469-1472.

16. Li UM, Lee BS, Shih CT, Lan WH, Lin CP. Cyclic Fatigue of Endodontic Nickel Titanium Rotary Instruments:
Static and Dynamic Tests. Journal of Endodontics 2002;28(6):448-451.

17. Hou X, Yahata Y, Hayashi Y, Ebihara A, Hanawa T, Suda H. Phase Transformation Behaviour and Bending Property of Twisted Nickel-Titanium Endodontic Instruments. International Endodntic Journal 2011;44( 3):253-258.

18. Alapati SB, Brantley WA, Iijima M, Clark WAT, Kovarik L, Buie C, Liu J,Ben johnson W. Metallurgical Characterization of a new Nickel-Titanium Wire for Rotary Endodontic Instruments. Journal of Endodontics 2009;35( 11):1589-1593.

19. Goo HJ, Kwak SW, Ha JH, Pedulla E, Kim HC. Mechanical Properties of Various Heat-treated Nickel-Titanium Rotary Instruments. Journal of Endodontics 2017;43( 11):1872-1877.

20. Shen Y, Zhou HM, Zheng YF, Peng B, Haapasalo M. Current Challenges and Concepts of the Thermomechanical Treatment of Nickel-Titanium Instruments. Journal of Endodontics 2013;39(2):163-172.

21. Santos LdA, Bahia MGdA, Las Casas EBd, Buono VTL. Comparison of the Mechanical Behavior Between Controlled Memory and Superelastic Nickel-Titanium Files via Finite Element Analysis. Journal of Endodontics 2013;39(11):1444-1447.

22. Gao Y, Gutmann Jl, Wilkinson K, Maxwell R, Ammon D. Evaluation of the Impact of raw Materials on the Fatigue and Mechanical Properties of ProFile Vortex Rotary Instruments. Jornal of Endodontics 2012;38( 3):398-401.

23. Zhang YQ, Jiang SY, Zhao YN, Tang M. Influence of Cooling Rate on Phase Transformation and Microstructure of Ti-50.9\%Ni Shape Memory Alloy. Transactions of Nonferrous Metals Society of China 2012;22(11):26852690 .

24. Oh SR, Chang SW, Lee Y, Gu Y, Son WJ, Lee W, Baek SH, Bae KS, Choi GW, Lim SM, Kum KY. A Comparison of Nickel-Titanium Rotary Instruments Manufactured Using Different Methods and Cross-Sectional Areas: Ability to Resist Cyclic Fatigue. Oral Surgery, Oral Medicine, Oral Pathology, Oral Radiology, and Endodontology 2010;109(4):622-628.

25. Elnaghy AM, Elsaka SE. Cyclic Fatigue Resistance of OneCurve, 2Shape, ProFile Vortex, Profile Vortex, Vortex Blue, and RaCe Nickel-Titanium Rotary Instruments in Single and Double Curvature Canals. Journal of Endodontics 2018;44(11):1725-1730. 